\title{
Efecto del crecimiento económico y de la complejidad legal sobre el Impuesto al Valor Agregado
}

\author{
Effect of economic growth and legal complexity on the Value Added Tax
}

\section{Javier Moreno Espinosa, Jaime Humberto Beltrán Godoy*, Leovardo Mata Mata}

\author{
Universidad Anáhuac, México \\ Recibido el 12 de octubre de 2018; aceptado el 27 de agosto de 2019 \\ Disponible en Internet el: 29 de agosto de 2019
}

\section{Resumen}

El objetivo es determinar el impacto del crecimiento económico y de la complejidad legal de la Ley del Impuesto al Valor Agregado (LIVA), en la recaudación del IVA para el periodo 1980 - 2016. Se utilizó un modelo de mínimos cuadrados ordinarios transformado mediante la metodología de Box - Cox. Los resultados muestran que existe una relación positiva entre el crecimiento económico y la recaudación del IVA y una relación negativa entre el índice de complejidad legal y la recaudación del IVA. Se recomienda extrapolar el análisis a otros impuestos para medir el impacto de sus reformas legales sobre su recaudación, se puede generar un efecto negativo por las modificaciones legales realizadas que podrían incrementar la complejidad de las leyes fiscales y reducir la comprensión de los sujetos obligados. En consenso con la literatura, se confirma un efecto negativo sobre la recaudación del IVA por el incremento en el índice de complejidad legal.

Código JEL: E62, C01, H30

Palabras clave: Impuestos; Crecimiento económico; Complejidad legal; IVA

*Autor para correspondencia

Correo electrónico jaime.beltrang@ anahuac.mx (J.H. Beltrán Godoy).

La revisión por pares es responsabilidad de la Universidad Nacional Autónoma de México. 


\begin{abstract}
This work is aimed to determine the impact of economic growth and the legal complexity of the Value-Added Tax Act (LIVA, in Spanish) in the VAT collection between 1980 and 2016. This analysis is based on an ordinary least squares model transformed using the Box-Cox methodology. The results reveal a positive relation between economic growth and VAT collection, and a negative relation between the legal complexity index and VAT collection. It is suggested to extrapolate this analysis of the effects of legal reforms on the collection of other taxes, because a negative effect may be generated by the modifications made by increasing the complexity of tax laws and reducing the understanding of the tax payers. In consensus with the existing literature, a negative effect on the VAT collection is confirmed by the increase in the legal complexity index.
\end{abstract}

JEL code: E62, C01, H30

Keywords: Taxes; Economic growth; Legal complexity, VAT

\title{
Introducción
}

Las decisiones de los contribuyentes de cumplir o no cumplir con sus obligaciones fiscales incluyendo las obligaciones de pago, pueden estar influenciadas por diferentes factores de índole económico y no económico. Como factores económicos se identifican al nivel de ingreso, las probabilidades de ser auditado, las auditorías fiscales, la tasa impositiva, los beneficios fiscales, las multas y las penalizaciones y como factores no económicos se identifican a las actitudes hacia los impuestos, las actitudes personales, la confianza en la autoridad fiscal y en el uso de los recursos y las normas sociales y nacionales establecidas (Barbuta, 2011).

Como factor económico de impacto en la recaudación del Impuesto al Valor Agregado (IVA), se consideró al Producto Interno Bruto (PIB), métrica relacionada con la actividad económica y reconocida en Naciones Unidas como el indicador más adecuado para medir crecimiento económico. Como factor no económico con influencia en la recaudación del IVA se consideró la complejidad legal de la Ley del Impuesto al Valor Agregado (LIVA), entendida ésta como la ambigüedad que se genera en el texto de la LIVA por las diferentes modificaciones legales realizadas y su consecuente influencia en la recaudación del impuesto.

Utilizar el PIB como indicador de la actividad económica es de interés analítico, porque de acuerdo con el Sistema de Cuentas Nacionales 2018 publicado por Naciones Unidas y adoptado por el Instituto Nacional de Estadística y Geografía (INEGI, 2018), éste se define como "el valor agregado de todas las unidades residentes más los impuestos sobre los productos (menos subvenciones a los productos); es decir, el valor agregado bruto a precios básicos se 
define como la producción valorada a precios básicos menos el consumo intermedio valorado a precios de comprador, más los impuestos menos las subvenciones sobre los productos".

Con base en esta definición el valor agregado debería estar relacionado en una proporción constante con el IVA, siempre y cuando la tasa impositiva a los productos fuera una sola y constante (Barro y Sala i Martin, 2009); sin embargo, la LIVA a lo largo de su historia ha establecido tasas diferenciadas para diversas actividades o sectores: $16 \%$ como tasa general, sin importar el acto o actividad ni la zona geográfica dentro del territorio nacional; actualmente el $8 \%$ como tasa en la frontera norte (antes 11\%) sin importar el acto o actividad económica; tasa $0 \%$ en alimentos y medicinas principalmente y exención a ciertas actividades económicas como el sector agropecuario. Por lo tanto, al no tener una tasa impositiva constante para todos los productos distorsiona el postulado teórico de proporcionalidad, motivo suficiente para analizar la relación entre el comportamiento del PIB y la recaudación del IVA.

Utilizar un índice de complejidad legal como factor no económico también genera interés relevante, porque de acuerdo con (Givati, 2009), la legislación fiscal es ambigua en muchos casos. A menudo existen diferentes interpretaciones de la Ley, lo que resulta en consecuencias fiscales sustancialmente diferentes. La complejidad inherente a la legislación fiscal y los cambios frecuentes en la Ley exacerban este problema.

Givati (2009) define como complejidad en un texto legal, a la incertidumbre que se genera por la presencia de ambigüedad en las interpretaciones de las disposiciones establecidas y clasifica como fuentes de ésta en el derecho tributario a tres aspectos: ambigüedad con respecto al significado preciso del lenguaje legal, ambigüedad con respecto a la aplicación de la Ley a una situación específica y ambigüedad con respecto al tipo de evidencia suficiente para establecer los hechos necesarios.

La presencia de la ambigüedad genera incertidumbre legal y genera problemas a los contribuyentes. Los cambios frecuentes en la Ley exacerban la generación de incertidumbre y disuade a algunos contribuyentes de cumplir con sus obligaciones fiscales al elevar los costos de cumplimiento.

$\mathrm{El}$ argumento relevante para estudiar la relación entre la complejidad legal de la LIVA y la recaudación del IVA; es que, a mayores modificaciones legales, se genera mayor ambigüedad y por ende mayor complejidad que a su vez incrementa los costos de cumplimiento que repercuten de forma negativa en la recaudación de impuestos.

La motivación para llevar a cabo la presente investigación es el hecho de que el IVA en México siendo la segunda fuente de ingresos de la Hacienda pública y a pesar de que se estableció desde 1980, su recaudación tributaria no ha rebasado el $4 \%$ como proporción del PIB, aún y cuando se han realizado modificaciones en la tasa impositiva. Este porcentaje se 
observa menor al contrastarse con el promedio de América Latina y los países de la Organización para la Cooperación y el Desarrollo Económicos (OCDE) quienes superan el 6.5\% y 6.9\% respectivamente (ITESM, 2013).

Por lo tanto, estudiar la relación y el impacto del (PIB) como factor económico y la complejidad legal de la LIVA como factor no económico sobre la recaudación del IVA busca contribuir a ampliar los resultados de investigaciones previas que estiman la elasticidad ingreso de la recaudación del IVA en función del comportamiento del PIB (Cárdenas, Ventosa y Gómez, 2008), (CEFP, 2009) y (Sacasa, 2012). Los resultados esperados consideran una relación positiva entre el PIB y la recaudación del IVA y una relación negativa entre la complejidad legal de la LIVA y la recaudación de este impuesto. En los siguientes apartados se presenta la revisión de literatura, las estimaciones, los resultados y las conclusiones.

\section{Revisión de Literatura}

En la literatura sobre crecimiento, las teorías neoclásicas y endógenas emplean modelos de crecimiento que discuten la influencia de los impuestos sobre el crecimiento económico. Este análisis se ha llevado a cabo desde dos perspectivas diferentes; se han estudiado las causas y la temporalidad existente entre el crecimiento y los impuestos.

En el enfoque de crecimiento neoclásico existen fuerzas exógenas, tales como el progreso tecnológico y las dinámicas poblacionales que causan un crecimiento constante. Los impuestos pueden ejercer solo una influencia temporal sobre la tasa de crecimiento durante las transiciones sucesivas hacia el crecimiento con equilibrio (Karagianni, Pempetzoglu y Saraidaris, 2013).

En los modelos de crecimiento endógeno, el crecimiento está determinado por el comportamiento de los agentes de la economía, la difusión del conocimiento entre productores y las externalidades positivas del capital humano contribuyen a evitar la tendencia a registrar rendimientos decrecientes en la acumulación de capital. Los impuestos que afectan a los determinantes del crecimiento como los rendimientos en la acumulación de capital o el volumen de inversión en investigación y desarrollo influyen permanentemente en el crecimiento económico (Barro y Sala i Martin, 2009).

En ambas teorías, existe una relación negativa implícita entre los impuestos y el crecimiento; sin embargo, esta relación no ha sido respaldada de manera concluyente por los hallazgos empíricos (Karagianni, Pempetzoglu y Saraidaris, 2013).

Existen estudios que abordan la relación entre impuestos y crecimiento y brindan evidencias mixtas entre estas variables. Koester y Kormendi (1989), Levine y Renelt (1992), Easterly y Rebelo (1993), Slemrod y Yitzhaki (1995), Mendoza et al. (1997) y Kneller et al. 
(1999) concluyeron que existe una relación positiva y en algunos casos una correlación poco significativa, entre el nivel promedio de impuestos y la producción en el corto y largo plazo.

King y Rebelo (1990), Barro (1991), Plosser (1992), Engen y Skinner (1992), Kormendi y Meguire (1995), Wright (1996) y Leibfritz et al. (1997) identificaron una correlación negativa entre los impuestos y el crecimiento económico.

De la Fuente (1997) analizó los efectos de la política fiscal sobre el crecimiento, específicamente los impactos que se generan por la composición y el nivel de gasto realizado por el gobierno. Concluyó que si el gasto total del gobierno como proporción del PIB se incrementa el crecimiento se reduce, mientras que un incremento en la inversión pública potencia el crecimiento económico.

Mankiw (2016) establece que la relación entre política fiscal y crecimiento económico se presenta en el sentido de que la política fiscal es un instrumento para lograr una expansión o una disminución de la actividad económica de un país. El mecanismo de transmisión de la política fiscal hacia la actividad económica se da mediante el uso de instrumentos tales como, las compras de gobierno o los impuestos.

El mecanismo de transmisión desde la demanda agregada, cuando el gobierno decide incrementar su gasto (política fiscal expansionista) aumenta la demanda agregada; alternativamente el gobierno puede disminuir impuestos lo que implica un aumento en el ingreso disponible que permite aumentar el consumo lo que también implica un aumento en la demanda agregada. Cuando se trata de una política fiscal contraccionista el gobierno disminuye su gasto y eso produce una disminución en la demanda agregada o alternativamente realiza un aumento de impuestos lo que genera una disminución del ingreso disponible, una disminución en consumo y una disminución en la demanda agregada.

Desde el lado de la oferta agregada la producción depende de los factores productivos; es decir, de la cantidad de capital, de la cantidad de trabajo y del nivel de tecnología. Para aumentar el nivel de producción en el largo plazo se deberá aumentar la cantidad de capital, mejorar el uso del factor trabajo o de la tecnología actual. Desde el punto de vista de Solow (1956) para elevar la cantidad de capital es necesario elevar la tasa de ahorro y de inversión en la economía, pero al mismo tiempo, para elevar el stock de capital se deberá reducir el consumo actual.

Para mejorar el uso del factor trabajo, la economía debe aprovechar de mejor forma su población económicamente activa disminuyendo la tasa natural de desempleo; sin embargo, las políticas que mejoran la eficiencia del trabajo, como son la educación o el aumento del progreso tecnológico, llevan a un uso más productivo del capital y del trabajo. Otra forma de mejorar la utilización del factor trabajo sería la reducción del desempleo, aunque esta ver- 
tiente puede tener costos altos, ya que para reducirlo se deberían disminuir las prestaciones de desempleo o bajar los salarios, pero estas medidas podrían perjudicar a las personas de menores ingresos y difícilmente reúne los consensos para que se lleven a cabo (Mankiw, 2016).

Por el lado de la oferta agregada o de la demanda agregada existen formas de elevar la producción en la economía mediante el uso de la política fiscal como instrumento para lograr un incremento del PIB. Este argumento teórico pone de manifiesto la relación entre la política fiscal y el crecimiento económico mediante el uso de sus instrumentos tales como el gasto de gobierno y los impuestos, éstos últimos con un impacto en la recaudación impositiva.

Investigaciones anteriores han demostrado que las estructuras fiscales tienen impacto en el crecimiento económico: Marsden (1983), demostró que un cambio en la política fiscal provocaría cambios en las decisiones y en la planeación económica de un país; Gober y Burns (1997) explicaron que las economías nacionales se encuentran altamente influenciadas por los cambios en los componentes de las estructuras fiscales. Ambos concluyeron que el comportamiento de la actividad económica se ve influenciada por la estructura y las decisiones en materia de política fiscal.

A pesar de la relación existente entre la política fiscal y el crecimiento económico generada a través del uso del instrumento fiscal denominado tasa impositiva, se ha demostrado que modificar las tasas particularmente en tiempos de recesión, podría causar cambios desfavorables tanto en el crecimiento económico, como en los niveles de recaudación. La curva de Laffer muestra la relación entre la tasa impositiva y el monto de recaudación de impuestos y establece que una tasa fiscal más alta no siempre genera una mayor recaudación; es decir, existe un umbral en el que se quiebra la relación que establece que a mayor tasa impositiva mayor recaudación (Parkin y Loria, 2015).

Hinrichs (1966) y Musgrave (1969) estudiaron la relación entre la proporción de ingresos fiscales y el PIB, concluyendo que dicha relación es nula en los países en vías de desarrollo.

De la revisión de la literatura se puede concluir que existe una relación positiva o negativa entre la política fiscal y el crecimiento económico cuando se busca expandir o contraer la producción; expansión o contracción que tiene efectos sobre la recaudación tributaria, relación que es parte del objetivo de estudio de la presente investigación al pretender explicar los efectos del crecimiento económico sobre la recaudación del Impuesto al Valor Agregado.

La literatura sugiere que la recaudación de impuestos se ve condicionada por diversos factores, entre ellos factores estructurales como el ingreso per cápita, el nivel de participación de la agricultura en el PIB y el nivel de apertura comercial; así como por otros factores, como la corrupción, la estabilidad política, la proporción de impuestos directos e indirectos, entre otros (Gupta, 2007). 
La Comisión Económica para América Latina y el Caribe (CEPAL, 2009), organismo dependiente de la Organización de las Naciones Unidas, identifica cinco determinantes directos de la recaudación tributaria: la legislación tributaria, el valor de la materia gravada, las normas de liquidación e ingreso de los tributos, el incumplimiento en el pago de las obligaciones fiscales entre otros factores.

Este organismo también identifica como determinantes de segundo grado, a los objetivos de la política económica y fiscal, las preferencias de los realizadores de la política tributaria, las variables de índole económica y la evaluación de costos y beneficios de las prácticas de evasión tributaria (CEPAL, 2009).

La Comisión Europea concluye que la recaudación del IVA se ve condicionada por su propia regulación (nivel impositivo, tasas diferenciadas, excepciones, etc.) y por otros factores macroeconómicos (crecimiento del PIB, nivel de consumo, importaciones, exportaciones, entre otros) (Bikas y Andruskaite, 2013).

En línea con (Bikas y Andruskaite, 2013) y de acuerdo con Barro y Sala i Martin (2009) la relación del PIB y la recaudación de impuestos, desde la óptica de la contabilidad del crecimiento se establece que cuando existe un impuesto sobre la producción o a las ventas, las empresas competitivas cumplirán con la condición de que la productividad marginal del trabajo y la productividad marginal del capital serán iguales al pago de los factores dividido por (1- $\tau)$, es decir:

$$
F_{L}=\frac{w}{(1-\tau)} ; \quad F_{K}=\frac{R}{(1-\tau)}
$$

Donde $F_{L}$ es la productividad marginal del trabajo y $F_{K}$ es la productividad marginal del capital, $w$ es el salario, $R$ es la renta del capital y $\tau$ es la tasa impositiva marginal sobre la producción; por lo tanto, si el impuesto sobre la producción (o a las ventas) es proporcional de forma que las tasas impositivas promedio y marginales son idénticas, la recaudación tributaria será igual a una proporción constante de la producción determinada por $(\tau Y)$.

La producción $Y$ será igual al pago a los factores más lo recaudado por el impuesto indirecto, entonces la producción será $Y=R k+w L+\tau Y$. Esto aplica cuando el impuesto sobre el valor añadido proporcional se aplica de igual forma al factor trabajo y al factor capital; es decir: $\tau_{K}=\tau_{L}$. Entonces si $\tau_{K}$ y $\tau_{L}$ son diferentes las ponderaciones sobre $K$ y $L$ también serán diferentes y la proporción $(\tau Y)$ ya no será constante (Barro y Sala i Martin, 2009).

Como se ha mencionado en párrafos anteriores, de acuerdo con el Sistema de Cuentas Nacionales Base 2013, el Valor Agregado Bruto de la economía se define como "el valor 
agregado de todas las unidades residentes más los impuestos sobre los productos (menos subvenciones a los productos)"; es decir, el valor agregado bruto a precios básicos se define como la producción valorada a precios básicos menos el consumo intermedio valorado a precios de comprador, más los impuestos menos las subvenciones sobre los productos. Con base en esta definición y de acuerdo con Barro y Sala i Martin (2009) el valor agregado debería estar relacionado en una proporción constante con el IVA; sin embargo, al existir tasas diferenciadas, tasa cero y las exenciones, el concepto teórico establecido por Barro y Sala i Martin (2009) y el Sistema de Cuentas Nacionales Base 2013 no se cumple y las distorsiones que se generan por la estructura fiscal actual del IVA son un argumento válido para analizar la relación y el impacto del crecimiento económico sobre la recaudación del IVA.

La relación del crecimiento económico con la recaudación del IVA para el caso de México ha sido estudiada por Cárdenas, Ventosa y Gómez (2008), el estudio se realizó con datos del primer trimestre de 1980 al segundo trimestre de 2005, las cifras fueron expresadas en pesos constantes de 2005, bajo un modelo de cointegración con orden de integración I(1) en niveles (series no estacionarias) e I(0) en primeras diferencias (series estacionarias). El orden de integración lo determinaron mediante la aplicación de la prueba Dicky - Fuller GLS sobre las series desestacionalizadas (Census X12-ARIMA). Concluyeron con un nivel de confianza del 5\% de que no hay pruebas suficientes para rechazar la existencia de raíz unitaria y con el mismo porcentaje de confianza rechazaron la hipótesis nula de raíz unitaria para todas las series en primeras diferencias. La elasticidad se estimó mediante un modelo en donde el IVA (variable dependiente) es el logaritmo de la recaudación en el periodo (t) y el ingreso es el logaritmo del PIB en el periodo (t) con variables de control dicotómicas que representan las modificaciones legislativas de la tasa impositiva de 1983 y 1992 como variables proxy de cambio estructural. Los resultados permitieron concluir que, la elasticidad ingreso a largo plazo de la recaudación del IVA respecto del PIB es de 1.12, lo que significa que, ante el aumento de un punto porcentual en el PIB, la recaudación a largo plazo del IVA se incrementa en $1.12 \%$.

El Centro de Estudios de las Finanzas Públicas (2009) con el objetivo de determinar el impacto del IVA en la planeación y formulación del presupuesto público, el monitoreo de la recaudación impositiva y la estimación del déficit público, estimó la elasticidad ingreso de la recaudación del IVA con respecto al PIB con datos del primer trimestre de 1980 al segundo trimestre de 2009, cifras que fueron expresadas en pesos constantes de 2003. Siguiendo los trabajos de (Cárdenas, Ventosa y Gómez, 2008), los datos se expresaron en logaritmos y fueron ajustados por estacionalidad (X-11 ARIMA). El orden de integración se determinó mediante la prueba aumentada de Dickey - Fuller (ADF) para identificar la existencia de 
raíz unitaria en las series tanto en nivel como en sus primeras diferencias; el resultado de esta prueba fue que las series son no estacionarias en nivel y estacionarias de orden uno en sus primeras diferencias. Las variables de control que se incorporaron en el modelo son las mismas que se trabajaron por (Cárdenas, Ventosa y Gómez, 2008). Los resultados de las estimaciones permitieron concluir que, ante un incremento de un punto porcentual en el PIB, la recaudación de IVA se incrementa en el largo plazo en 1.88\%. El CEFP (2009), explicó que las diferencias en los resultados respecto de los trabajos de (Cárdenas, Ventosa y Gómez, 2008) se explican principalmente por la diferencia en los periodos de análisis ya que las variables de control en ambos trabajos siguieron la misma metodología y mencionaron que en (Cárdenas, Ventosa y Gómez, 2008) se reconoce que la elasticidad ingreso del IVA respecto del PIB es relativamente baja.

El Programa de Naciones Unidas para el Desarrollo (Sacasa, 2012) también estudió la relación del IVA con el PIB para el periodo del tercer trimestre de 1981 al cuarto trimestre de 2009, con cifras expresadas a pesos constantes de 2003, bajo un modelo en el cual se considera la recaudación del IVA como una función del nivel de ingreso, medido a través del PIB. La estimación de la elasticidad se realizó bajo el procedimiento de Johansen (1988), atendiendo la presencia de series no estacionarias, para obtener un vector de cointegración, el cual mediante el teorema de Engle y Granger (1987) el vector de cointegración se consideró como un mecanismo de corrección de errores. Por lo tanto, dado que el PIB y la recaudación del IVA son series no estacionarias I(1), se especificó un modelo de cointegración que utilizó el procedimiento de Johansen (1988) para su estimación. Normalizando el vector de cointegración se concluyó que existe una relación estable a largo plazo entre la recaudación del IVA con el PIB y los resultados concluyeron que "ante el aumento de un punto porcentual en el PIB, la recaudación a largo plazo del IVA se incrementa 1.28\% (Sacasa, 2012).

Los trabajos de Cárdenas, Ventosa y Gómez (2008), del Centro de Estudios de las Finanzas Públicas (CEFP, 2009) y del PNUD (Sacasa, 2012) permiten concluir que es factible estimar la elasticidad ingreso entre el crecimiento económico y la recaudación del Impuesto al Valor Agregado (IVA). Los tres estudios identificados en la literatura siguieron la metodología de cointegración y sus resultados facilitan la conclusión de que la relación entre ambas variables es positiva y ante movimientos en el PIB se generan movimientos en la recaudación del impuesto. 
Tabla 1

Principales características de los estudios que analizan la relación del PIB y la recaudación del IVA

\begin{tabular}{cccc}
\hline Concepto & $\begin{array}{c}\text { Cárdenas, Ventosa } \\
\text { y Gómez (2008) }\end{array}$ & CEFP (2009) & PNUD (2012) \\
\hline Elasticidad & 1.12 & 1.88 & 1.28 \\
$R^{2}$ & 0.93 & 0.96 & nd \\
$\begin{array}{c}\text { Periodo } \\
\text { de estudio }\end{array}$ & 1T1980-2T2005 & 1T1980-2T2009 & 3T1981-4T2009 \\
Datos & Pesos constantes 2005 & Pesos constantes 2003 & Pesos constantes 2003 \\
Método & Cointegración & Cointegración & Cointegración \\
de estimación & Dickey - Fuller & \\
& Dickey - Fuller aumentada (El- & aumentada (Elliot, \\
Pruebas & Rothenberg y Stock, & Johansen (1988) \\
& & 1996) & \\
\hline
\end{tabular}

Fuente: Cárdenas, Ventosa y Gómez (2008), CEFP (2009) y Sacasa (2012).

En la tabla 1 se expresan las principales características de los tres estudios que analizan la relación entre el PIB y la recaudación del IVA.

Además de la condicionante que ejerce el crecimiento económico sobre la recaudación de impuestos, existen otros factores que influyen en el cumplimiento de las obligaciones tributarias por parte de los contribuyentes, factores que pueden generar efectos, positivos o negativos, sobre la recaudación, por su influencia en el comportamiento y en las decisiones de los contribuyentes (OCDE, 2004).

La CEPAL (2009), Gupta (2007), Barbuta (2011) y Helhel y Ahmed (2014), han establecido algunas clasificaciones de los factores que condicionan la recaudación tributaria, estas clasificaciones establecen que los factores pueden ser de índole económico y no económico o también de índole interno y externo.

Barbuta (2011) clasificó a los factores como económicos y no económicos. Entre los económicos, como se ha expresado en párrafos anteriores, destaca al nivel de ingreso, las tasas impositivas, los beneficios fiscales establecidos en la estructura fiscal, las probabilidades de ser objeto de auditoría y las multas o sanciones por omitir el pago (parcial o total) de impuestos. 
Los factores no económicos que influyen en el cumplimiento tributario son: la percepción de los contribuyentes acerca de los impuestos; las normas personales, sociales y nacionales y la percepción acerca de la justicia del sistema fiscal relacionada con la justicia distributiva.

Helhel y Ahmed (2014) clasificaron a los factores que impactan el cumplimiento tributario en dos grupos: internos y externos. En los determinantes internos se encuentran la forma de pensar y la percepción de los contribuyentes acerca de los impuestos; en los determinantes externos, están el sistema fiscal, la actividad de las autoridades tributarias con respecto a los contribuyentes y las disposiciones legales.

Estas clasificaciones resultan importantes, debido a que amplían el universo de factores que pueden ejercer una influencia sobre la recaudación del IVA, además del ingreso. A partir de estas clasificaciones, la presente investigación busca ampliar el estudio de los factores que impactan la recaudación del IVA y considera además del PIB la influencia que ejerce la complejidad legal de la Ley del IVA a través del índice de complejidad legal generado en los trabajos de (Moreno, Beltrán y Mata, 2017), de tal forma que se analice de forma conjunta el impacto del PIB y la complejidad legal sobre la recaudación del IVA.

El estudio de la complejidad legal es un tema relativamente nuevo si se contrasta con los estudios de los aspectos económicos que tienen su origen en la Riqueza de las Naciones de Adam Smith en 1776; los antecedentes primigenios del análisis de la complejidad legal se registran a partir de los trabajos realizados en 1972 por El Colegio de Abogados de EE.UU. (American Bar Association, - ABA, por sus siglas en inglés) que consistieron en el análisis del texto legal del Código Fiscal de los Estados Unidos.

A partir de los trabajos del Colegio de Abogados de EE. UU. y hasta la primera década del siglo XXI, el tema de la complejidad legal y las técnicas de minería de texto, emergen como una práctica para la medición de la evolución de los diferentes textos legales, siendo la investigación en el área fiscal la de mayor relevancia debido a sus implicaciones en la relación entre el Estado y los contribuyentes (ABA, 2018).

Diver (1983) en sus estudios sobre la precisión optima de las reglas administrativas concluyó que un texto se considera complejo cuando éste presenta un bajo nivel en tres elementos: transparencia, accesibilidad y congruencia; por lo tanto, de acuerdo con este autor, una Ley es más compleja en tanto presente menos de estos tres elementos.

Schuck (1992), fue de los primeros autores en abordar el tema de la complejidad legal, definió a un sistema complejo "cuando sus reglas, procesos e instituciones presentan cuatro características: densidad, tecnicismos, diferenciación e incertidumbre” y destacó que aún y cuando su investigación fue uno de los primeros trabajos acerca de la complejidad legal, ya se 
identificaba a la Ley fiscal como una Ley con un alto grado de complejidad, por la presencia de estos cuatro elementos.

Kades (1997) afirmó que el mejor ejemplo de complejidad legal se ubica en la Ley fiscal, a pesar de que hacía referencia a la Ley Federal de Ingresos Fiscales de Estados Unidos, dicha afirmación es útil para demostrar la existencia de complejidad en las leyes fiscales. La primera definición que utilizó en cuanto a complejidad fue la intención de las sociedades en regular todas las interacciones humanas, intención que en lugar de simplificar complica los textos legales que regulan las relaciones humanas. Retomó los argumentos de Diver (1983) en cuanto a que un texto legal es más complejo cuando presenta menos transparencia, es poco accesible y muestra poca congruencia o mayor ambigüedad.

Surrey (1969), autor de diversos estudios sobre el sistema fiscal estadounidense, indicó que "la complejidad es resultado del hecho de que las complicadas leyes fiscales son aplicables en un sistema económico y legal muy complejo; de manera natural, la sociedad crea y demanda un sistema legal complejo". Su argumento se ubica en la misma línea que Diver (1983) y Kades (1997) en el sentido de que al buscar reglamentar todas y cada una de las interacciones humanas, las normas se convierten en textos complejos.

Katz y Bommarito (2014), autores reconocidos por sus aportaciones a la medición de la complejidad legal, en sus primeras investigaciones generaron un indicador que tomaba como base el número de páginas en un texto, sin ir más allá que el simple conteo de palabras. Como consecuencia no se tenían indicadores para determinar si alguna regulación o cualquier otro texto con efectos legales era más o menos complejo a partir de las modificaciones que se realizaban.

Para atender la necesidad de medir la complejidad legal en un texto Katz y Bommarito (2014) desarrollaron un indicador que integra tres componentes: la estructura, el lenguaje y la interdependencia de un texto legal.

Para medir la estructura de una Ley, los autores consideraron que el texto de un documento legal tiene una estructura semejante a una red jerárquica o a una estructura de un árbol; la profundidad de cada uno de los elementos representa las ramas del árbol hasta llegar al más mínimo detalle, lo que culminaría en un diagrama de árbol de decisiones.

Para medir el concepto de lenguaje, los autores propusieron que los textos de dicho árbol, que van de lo general a lo particular, en la estructura de una Ley, están representados por palabras, mismas que conforman el lenguaje y que pueden tener diferentes longitudes y significados.

Para medir interdependencia de una Ley, consideraron las referencias contenidas en el texto de una Ley hacia otros textos dentro de la misma Ley o a otros textos de leyes diferentes, de 
tal manera que para lograr la comprensión de un texto legal habría que revisar o comprender simultáneamente textos legales dentro del texto de la propia Ley analizada o incluso de otras leyes adicionales a las que se hace referencia.

Ruhl y Katz (2015) publicaron una investigación en la que midieron la complejidad legal del sistema fiscal estadounidense; dichos autores concluyeron que un sistema legal es más complejo cuando existe un mayor grado de interdependencia entre sus componentes; es decir, en un sistema legal simple, el modificar o eliminar algún elemento, no alteraría en gran medida el funcionamiento de dicho sistema, porque se presenta una baja interrelación entre los textos de las diferentes leyes, no así en sistemas complejos, que cuando se modifica algún ordenamiento se tienen que ajustar todos los textos relacionados con dicha modificación.

En México, el Centro de Estudios Económicos del Sector Privado (CEESP, 2018) -organismo dependiente el Consejo Coordinador Empresarial-indicó que el país tiene un sistema fiscal complejo y costoso de administrar, que estimula la evasión y la elusión fiscal debido a la multiplicidad de impuestos, exenciones, tasas diferenciadas e impuestos especiales.

La Procuraduría de la Defensa del Contribuyente (Prodecon) explica que los contribuyentes en México enfrentan dificultades para cumplir con las obligaciones que la Ley establece, debido a la excesiva y compleja regulación que dificulta la determinación de las contribuciones, implicando altos costos y constituyéndose en un factor de desaliento en el cumplimiento (Bernal, Padilla y Pérez, 2011).

A partir de la revisión de la literatura, se detectó que existe una relación entre la recaudación del IVA y algunos factores económicos y no económicos, tales como el crecimiento económico y la complejidad legal de la Ley del IVA. En el caso del PIB y la recaudación del IVA, la hipótesis de trabajo es que en periodos de mayor crecimiento económico habrá mayor recaudación de IVA y en el caso de la recaudación del IVA y la complejidad legal de la Ley del IVA los resultados esperados son que en periodos con mayor complejidad legal habrá menos recaudación de IVA.

La motivación de estudiar estas relaciones es que el IVA representa la segunda fuente de ingresos de la Hacienda Pública y desde su entrada en vigor en 1980, su recaudación no ha rebasado el $4 \%$ como proporción del PIB, a pesar de que se han realizado variaciones en la tasa impositiva.

Para el estudio de estas relaciones, a continuación, se presenta la metodología y los resultados de las pruebas que permiten medir el impacto del PIB y la complejidad legal de la Ley del IVA sobre la recaudación del Impuesto al Valor Agregado. 


\section{Metodología}

La estimación que se realizó en esta investigación tomó como antecedente los trabajos de Cárdenas, Ventosa y Gómez (2008) y Katz y Bommarito (2014). El primero se utilizó para estimar la elasticidad de la relación entre la recaudación del IVA y el crecimiento económico; el segundo se utilizó para estimar la elasticidad de la relación entre la recaudación del IVA y la complejidad legal de la Ley del IVA.

El modelo que se emplea se realizó bajo una transformación con el método de Box-Cox, en primera instancia para buscar un método alternativo a los trabajos realizados sobre la relación de estas dos variables y en segundo lugar por las siguientes razones técnicas: permite corregir problemas de heterocedasticidad, no normalidad y no estacionariedad sin recurrir a modelos de corrección de errores, $\operatorname{ARIMA}(\mathrm{p}, \mathrm{q}) \mathrm{u}$ otros, donde la interpretación del efecto marginal no es directa (Porunov, 2010). Como variables de control se incorporaron variables proxy cuyo fundamento es el cambio legislativo que modifica la tasa impositiva del IVA y se considera de acuerdo con los trabajos de (Cárdenas, Ventosa y Gómez, 2008) como variables que simulan cambio estructural durante la vigencia del impuesto y que han afectado su recaudación.

Como aportación de esta investigación a los trabajos realizados sobre los determinantes económicos que afectan la recaudación del IVA, se integró al modelo como variable explicativa el indicador de complejidad legal estimado por (Moreno, Beltrán y Mata, 2017). Este indicador de complejidad legal tomó como base los trabajos realizados por Katz y Bommarito (2014).

El indicador de complejidad legal de la LIVA, propuesto en esta investigación, considera a la estructura de la Ley como la forma en que está organizado el texto legal y a partir del cual se identifica la profundidad de los elementos que la integran; es decir, se asigna un ponderador numérico mayor conforme aumenta la profundidad de cada uno de los elementos de la Ley; los valores van del 1 al 5 dependiendo de si se trata de un artículo, párrafo, fracción, inciso y numeral respectivamente, de tal manera que se pueda identificar los momentos en que se han realizado adiciones o derogaciones al texto de la Ley del IVA.

El componente de lenguaje, a diferencia del modelo propuesto por (Katz y Bommarito, 2014) se analizó desde la óptica de entropía $(S)$, con el objeto de determinar las veces que cada palabra se repite en el texto como un indicador de la frecuencia relativa. La estimación de la frecuencia relativa se realizó mediante el uso de técnicas de minería de texto en cada uno de los textos publicados de la Ley del IVA desde 1978 hasta 2016. La estimación de la entropía muestra que cuando un texto es uniforme su entropía es cero y cuando este valor es cercano a uno se trata de un texto poco uniforme. 
El componente de interdependencia, también se ajustó respecto de lo estipulado por Katz y Bommarito (2014) y se analizó considerando las referencias internas y externas en el texto de la Ley; es decir, se consideraron cuatro tipos de referencias: cuando se refiere a algún párrafo anterior; a algún elemento dentro de la Ley del IVA; a algún elemento específico de otra Ley y alguna referencia general a otra Ley. Los ponderadores para calcular la interdependencia se asignaron bajo el supuesto de que es más sencilla la comprensión de una referencia al párrafo anterior que cuando se cita algún texto genérico a otra Ley distinta del IVA; entonces, los valores de los ponderadores van del 1 al 4 asignado el número uno a la referencia más sencilla que es al párrafo anterior y así sucesivamente.

El indicador de complejidad legal conjunto se determinó mediante la suma de los valores obtenidos para cada uno de los componentes (estructura, lenguaje e interdependencia) de tal forma que se obtuvo un valor para cada uno de los trimestres del periodo de 1980 a 2016 y este valor se incorporó al modelo propuesto en esta investigación para el cálculo de la elasticidad de la recaudación del IVA respecto de la complejidad legal de la Ley del IVA.

Para llevar a cabo esta estimación, se utilizaron cifras trimestrales anualizadas a precios constantes de 2013, desestacionalizadas (X-13 ARIMA) para el periodo de 1980 a 2016 sobre el IVA y el PIB y para el indicador de complejidad legal se utilizó la estimación realizada por (Moreno, Beltrán y Mata, 2017) que conjunta en un indicador trimestral los valores de estructura, lenguaje e interdependencia.

Es oportuno mencionar que el indicador de complejidad legal que se utiliza en la estimación de la elasticidad de la recaudación del IVA presenta ventajas y limitaciones. En primer lugar, considera diversos ponderadores para incorporar la evolución del texto LIVA en tres indicadores de complejidad, según la metodología (Katz y Bommarito, 2014), lo cual no se ha encontrado hasta el momento en la literatura para el caso de México. Como limitante se tiene el tiempo de acopio del universo de textos legales publicados a comparar, además del esfuerzo de cómputo que se requiere para la aplicación de la minería de texto.

Con base en estas definiciones y las cifras utilizadas el modelo es una especificación de regresión múltiple con logaritmo natural en las variables dependiente e independientes. De esa manera, los coeficientes estimados se pueden interpretar como una elasticidad entre las variables (Gujarati y Porter, 2010):

$$
\ln \left(I V A_{t}\right)=\alpha_{0}+\alpha_{1} \ln \left(P I B_{t}\right)+\alpha_{2} \ln \left(C_{t}\right)+u_{t}
$$


Donde:

$I V A t=$ Recaudación de IVA, a precios constantes, base 2013 (millones de pesos).

$P I B t=$ PIB a precios constantes, base 2013 (millones de pesos).

$C_{t}=$ Índice de complejidad legal.

$u_{t}=$ Perturbación aleatoria.

El modelo original (ecuación 1) presenta dificultades en su estimación, ya que las series económicas de IVA y PIB son series no estacionarias y presentan correlación positiva (Cárdenas, Ventosa y Gómez, 2008), lo cual viola los supuestos básicos del modelo de mínimos cuadrados ordinarios, implica una regresión espuria y por ende arroja estimadores no consistentes (Greene, 2018).

Para corregir estos problemas y asegurar que se cumple con los supuestos del modelo de mínimos cuadrados ordinarios, se puede emplear la transformación Box y Cox sobre el logaritmo natural de la recaudación del IVA. Este procedimiento permite transformar la variable dependiente de un modelo de regresión de tal manera que los residuales se comporten como una normal con media cero y varianza constante (Porunov, 2010).

El método de Box y Cox modifica la variable dependiente $\ln \left(I V A_{t}\right)$ del modelo de regresión mediante la elección óptima de un parámetro $\theta$ tal que la ecuación (2) satisface que $v_{t} \sim N\left(0, \sigma^{2}\right)$, lo cual corrige el problema de heterocedasticidad, no normalidad y no estacionariedad (Vélez, Correa y Marmolejo-Ramos, 2015).

$$
\left[\ln \left(I V A_{t}\right)\right]^{\theta}=\beta_{0}+\beta_{1} \ln \left(P I B_{t}\right)+\beta_{2} \ln \left(C_{t}\right)+v_{t}
$$

En la expresión (2), la variable $[\ln (I V A)]^{\theta}$ es la transformación del logaritmo natural de la recaudación de IVA bajo el parámetro $\theta$, a precios constantes.

Por otra parte, es importante señalar que la familia de transformaciones propuesta por Box y Cox (1964) para una variable dependiente $y(\theta)$ es:

$$
\begin{gathered}
y(\theta)= \begin{cases}\frac{(y+m)^{\theta}-1}{\theta} ; & \theta \neq 0 \\
\ln (y+m): & \theta=0\end{cases} \\
m=0 \text { si } y>0
\end{gathered}
$$

Donde la función de máxima verosimilitud para la regresión $y(\theta)=X \beta+u$ es:

$$
f(y)=\frac{1}{\left(\sqrt{2 \pi \sigma^{2}}\right.} e^{-\frac{(y(\theta)-X \beta)^{\prime}(y(\theta)-X \beta)}{2 \sigma^{2}}} J(\theta, y)
$$


Además, el Jacobiano $J(\theta, y)$ está dado por:

$$
J(\theta, y)=\prod_{i=1}^{n} \frac{\partial y_{i}(\theta)}{\partial y_{i}}=\prod_{i=1}^{n} y_{i}(\theta-1)
$$

Bajo este enfoque se obtienen los estimadores de máxima verosimilitud de $\beta$ y $\sigma^{2}$, los cuales están dados por:

$$
\begin{gathered}
\hat{\beta}(\theta)=\left(X^{\prime} X\right)^{-1} X Y(\theta) \\
\hat{\sigma}^{2}(\theta)=Y(\theta)(I-P) Y(\theta) / n
\end{gathered}
$$

Siendo que el valor óptimo de se obtiene simultáneamente bajo máxima verosimilitud (Vélez, Correa y Marmolejo-Ramos, 2015).

Para estimar el modelo propuesto en la ecuación (2), se pueden añadir variables de control dicotómicas $d_{k}$ para delimitar e incorporar $k$ cambios estructurales que haya presentado la ley del IVA a lo largo de los años 1980-2016, similar a lo realizado en (Cárdenas, Ventosa y Gómez, 2008).

En ese caso, la ecuación resultante sería:

$$
\left[\ln \left(I V A_{t}\right)\right]^{\theta}=\beta_{0}+\beta_{1} \ln \left(P I B_{t}\right)+\beta_{2} \ln \left(C_{t}\right)+\sum_{k=1}^{m} \varphi_{k} d_{k}+v_{t}
$$

En la especificación (3) es factible que se presenten problemas de autocorrelación debido al carácter temporal de las variables, de ahí que se utilice el procedimiento de Prais-Winsten y Cochrane-Orcutt iterativos para solventar dicho problema y obtener efectos marginales robustos entre las variables independientes y la variable dependiente (Kristoufek, Janda y Zilberman, 2014).

Concretamente, el procedimiento de Prais-Winsten y Cochrane-Orcutt reestiman iterativamente un modelo de regresión bajo el esquema de una especificación $A R(p)$ para el término aleatorio, el cual está dado por $v_{t}=\phi_{1} v_{t-1}+\phi_{2} v_{t-2}+\cdots+\phi_{p} v_{t-p}$ para algún $p \geq 1$. El procedimiento comienza con un punto inicial $\hat{\phi}_{i}$ para los coeficientes $i=1,2, \ldots, p$ y donde se espera alcanzar convergencia (Greene, 2018).

En el caso de la ecuación (3), el modelo transformado sería:

$$
\begin{gathered}
Y_{t}^{*}=Y_{t}-\phi_{1} Y_{t-1}+\phi_{2} Y_{t-2}+\cdots+\phi_{p} Y_{t-p} \\
X_{j, t}^{*}=X_{j, t}-\phi_{1} X_{j, t-1}+\phi_{2} X_{j, t-2}+\cdots+\phi_{p} X_{j, t-p}
\end{gathered}
$$


Donde $Y_{t}^{*}$ es la variable dependiente y $X_{j, t}^{*}$ son las variables explicativas, de tal manera que se obtiene la ecuación auxiliar:

$$
\left\{\left[\ln \left(I V A_{t}\right)\right]^{\theta}\right\}^{*}=\beta_{0}+\beta_{1}\left\{\ln \left(P I B_{t}\right)\right\}^{*}+\beta_{2}\left\{\ln \left(C_{t}\right)\right\}^{*}+\sum_{k=1}^{m} \varphi_{k} d_{k}^{*}+\varepsilon_{t}
$$

Donde $\varepsilon_{t}$ es un proceso de ruido blanco. A este respecto, se emplean las pruebas de hipótesis de Breusch-Pagan y Ljung-Box para corroborar la corrección por autocorrelación y determinar el orden para el modelo AR(p).

Es importante señalar que la metodología anterior se sustenta como procedimiento alternativo a la cointegración u otras técnicas utilizadas por diversos autores. Específicamente, Sakia (1992) estudió la transformación propuesta por Box y Cox (1964) y propuso que para realizar el análisis de datos se debe generar un nuevo modelo a partir de los datos originales, que contenga las características principales del fenómeno a estudiar mediante la realización de una transformación a los datos, de tal forma que permita cumplir con los supuestos de independencia, normalidad y varianza constante. Sus conclusiones fueron que la transformación de Box y Cox, además de ser ampliamente utilizada desde que los autores la propusieron en 1964, ha encontrado una amplia utilidad en la determinación de relaciones funcionales en el campo de la econometría.

Castaño (2011) partió del hecho de que en modelos econométricos la utilización del análisis de regresión lineal clásico se basa en el comportamiento de los errores de forma aditiva, con distribución normal y con varianza constante. Sin embargo, estos supuestos no se cumplen cuando existen observaciones atípicas en los datos. Sus conclusiones fueron que el procedimiento Box y Cox es sensible cuando existen datos atípicos en la variable dependiente; por lo tanto, cuando no existen datos atípicos el procedimiento de Box y Cox es eficiente, aún en el caso de muestras pequeñas.

Porunov (2010) justifica que la transformación de Box-Cox es una alternativa a las técnicas tradicionales, tales como los mínimos cuadrados generalizados, la metodología de Box-Jenkins o los modelos de corrección de errores (cointegración) para enfrentar la violación de los supuestos del modelo de regresión clásico de no normalidad, heterocedasticidad o no estacionariedad.

En este trabajo se sigue este enfoque alternativo, ya que en la literatura se encuentran investigaciones con las variables de IVA y PIB como (Cárdenas, Ventosa y Gómez, 2008), donde se han empleado los métodos usuales. Es decir, el espíritu de este artículo es extender sus resultados con la finalidad de tener un marco comparativo, en relación con la inclusión de la variable complejidad. 


\section{Estimaciones y resultados}

En este apartado se presentan las estimaciones y los resultados del modelo transformado (ecuación 3.1) de acuerdo con la metodología enunciada en el apartado anterior. Para ello, primero se verifica que las series de tiempo no sean estacionarias en niveles, estacionarias en primera diferencia y que los residuales de la ecuación (1) no cumplen el supuesto de normalidad, homocedasticidad y ausencia de autocorrelación. De ahí que la transformación BoxCox sea una herramienta pertinente, en conjunto con el procedimiento de Cochrane-Orcutt.

Tabla 2a

Violación de supuestos del modelo de regresión en ecuación (1)

\begin{tabular}{|c|c|c|c|}
\hline \multirow{2}{*}{ Test } & \multicolumn{3}{|c|}{ Variables en niveles } \\
\hline & $\ln \left(I V A_{t}\right)$ & $\ln \left(P I B_{t}\right)$ & $\ln \left(C_{t}\right)$ \\
\hline DFA & $0.170(0.9420)$ & $1.746(0.9982)$ & $1.310(0.6248)$ \\
\hline PP & $0.695(0.9876)$ & $3.245(1.000)$ & $1.691(0.6247)$ \\
\hline KPSS & $3.30(0.000)$ & $1.44(0.000)$ & $1.50(0.000)$ \\
\hline \multirow{2}{*}{ Test } & \multicolumn{3}{|c|}{ Variables en primera diferencia } \\
\hline & $\ln \left(I V A_{t}\right)$ & $\ln (P I B t)$ & $\ln \left(C_{t}\right)$ \\
\hline DFA & $19.498(0.000)$ & $17.265(0.000)$ & $12.514(0.000)$ \\
\hline PP & $21.075(0.000)$ & $16.144(0.000)$ & $12.512(0.000)$ \\
\hline KPSS & $0.013(0.1234)$ & $0.042(0.1576)$ & $0.014(0.1618)$ \\
\hline
\end{tabular}

Fuente: elaboración propia.

En la tabla 2a se puede observar que las series de tiempo del modelo especificado no son estacionarias en niveles, pero sí en primeras diferencias, como se reporta en (Cárdenas, Ventosa y Gómez, 2008), (CEFP, 2009) y (Sacasa, 2012).

En la tabla $2 \mathrm{~b}$ se muestra que los residuales de la regresión de la ecuación (1) presentan problemas de heterocedasticidad, normalidad y correlación serial, pues en cada caso se rechaza la hipótesis nula. No obstante, no existen problemas de multicolinealidad, pues el estadístico VIF es menor a diez. 
Tabla $2 b$

Violación de supuestos del modelo de regresión en ecuación (1)

\begin{tabular}{cll}
\hline \multirow{2}{*}{ Test } & & \\
\cline { 2 - 3 } & \multicolumn{1}{c}{ Estadístico de prueba } & Valor $\mathrm{p}$ \\
\hline White & 48.315 & 0.000 \\
\cline { 2 - 3 } Breusch-Godfrey (1-3 rezagos) & 49.866 & 0.000 \\
\cline { 2 - 3 } Jarque-Bera & 20.213 & 0.000 \\
\hline VIF & 1.96 & NA \\
\hline
\end{tabular}

Fuente: elaboración propia.

Dado los resultados de la tabla 2 b se procede a la transformación de Box-Cox, para lo cual se tuvo que calcular el valor del parámetro $\theta$, con el objetivo de normalizar los residuales de la ecuación (1), permitiendo así la corrección de los problemas descritos para el modelo original de mínimos cuadrados ordinarios.

Tabla 3

Estimación del parámetro $\theta$ para la transformación Box-Cox

\begin{tabular}{ccccccc}
\hline $\ln (\mathrm{IVA})$ & Coeficiente & $\begin{array}{c}\text { Error } \\
\text { Estándar }\end{array}$ & Estadístico z & Valor $\mathrm{p}$ & \multicolumn{2}{c}{$\begin{array}{c}\text { Intervalo de confianza } \\
(95 \%)\end{array}$} \\
$\theta$ & 3.6215 & 0.1244 & 29.12 & 0 & 3.3778 & 3.8653 \\
\hline
\end{tabular}

Fuente: elaboración propia.

En la tabla 3 se muestra la estimación del parámetro $\theta$ que corresponde a la transformación de la variable dependiente $\ln (I V A)$. El estimador de $\theta$ es significativo a un nivel de confianza superior al $95 \%$.

Posterior, de la estimación del parámetro $\theta$ se estimó el modelo de regresión con la transformación Box-Cox mediante máxima verosimilitud para encontrar los estimadores robustos y que se satisfaga la condición de que la perturbación aleatoria sea $N\left(0, \sigma^{2}\right)$. Sin embargo, dado el problema de correlación serial se aplicó también el procedimiento de Cochrane-Orcutt para estimar el modelo de la ecuación (3.1). 
Tabla 4

Estimación de los parámetros del modelo

\begin{tabular}{lllll}
\hline $\ln ($ IVA $)$ & Efecto marginal & Error estándar & Estadístico t & Valor $\mathrm{p}$ \\
\hline $\ln (\mathrm{C})$ & -1.139 & 0.487 & -2.339 & 0.010 \\
$\ln (\mathrm{PIB})$ & 4.510 & 0.661 & 6.825 & 0.000 \\
\hline
\end{tabular}

\begin{tabular}{ll}
\hline Prueba de raíz unitaria sobre los residuales \\
\hline Estadístico $\mathrm{t}$ & -2.345 \\
Valor $\mathrm{p}$ & 0.0932 \\
\hline
\end{tabular}

Prueba de heterocedasticidad (White)

\begin{tabular}{ll}
\hline Estadístico chi2 & 2.73 \\
Valor $p$ & 0.1183 \\
\hline
\end{tabular}

\begin{tabular}{lc}
\hline \multicolumn{2}{l}{ Prueba de correlación serial (Breusch-Godfrey, 4 rezagos) } \\
\hline Estadístico chi2 & 1.673 \\
Valor $p$ & 0.1959 \\
\hline
\end{tabular}

\begin{tabular}{ll}
\hline \multicolumn{2}{l}{ Prueba de correlación serial (Ljung-Box, 4 rezagos) } \\
\hline Estadístico chi2 & 0.2948 \\
Valor $p$ & 0.5872 \\
\hline
\end{tabular}

\begin{tabular}{ll}
\hline Prueba de Jarque-Bera & \\
\hline Estadístico chi2 & 2.742 \\
Valor $p$ & 0.2538 \\
\hline
\end{tabular}

Fuente: elaboración propia.

Los efectos marginales para el modelo Box-Cox, en conjunto con el procedimiento de Cochrane-Orcutt, se muestran en la tabla 4, donde se puede observar que a mayor complejidad legal menor recaudación del IVA. El coeficiente negativo (-1.139) se puede interpretar como una elasticidad de largo plazo entre las variables. Asimismo, el coeficiente positivo (4.510) relaciona positivamente al IVA con la variable PIB y se puede estudiar análogamente como una elasticidad. Los signos corresponden al marco teórico que se ha revisado y concuerdan con lo esperado (Katz y Bommarito, 2014) y (Cárdenas, Ventosa y Gómez, 2008). 
En relación con el comportamiento de los residuales, en la tabla 4 se muestra que constituyen una serie de tiempo estacionaria y no existen problemas de heterocedasticidad ni correlación serial, pues el valor p es mayor a 5\%. Asimismo, se cumple el supuesto de normalidad, bajo la prueba Jarque-Bera.

De igual forma, en la tabla 5, se muestra la estimación de los parámetros de las variables dicotómicas, variables de control, que indican cambios estructurales en el periodo de 1980 a 2016 y que están dados por el cambio de la tasa impositiva del IVA en los años que se llevaron a cabo. Como se puede observar, el cambio en la tasa impositiva tiene efectos positivos o negativos en la recaudación del IVA, dependiendo de si el cambio en la tasa es al alza o a la baja.

Respecto de la eliminación de la variable de control d1995 por su no significancia estadística, se determinó no eliminarla por la siguiente razón: económicamente tiene relevancia mantenerla dentro de la estimación debido a que en 1995 se registró la mayor caída en el PIB desde los años treinta y a pesar de que se aumentó la tasa impositiva del 10\% al 15\% para fortalecer las finanzas públicas, los efectos en la recaudación del IVA de ese año fueron poco significativos ya que se alcanzó una cifra del 2.2\% como proporción del PIB, cifra muy similar a la registrada en 1994 con una tasa impositiva del 10\%, comportamiento que puede justificar la no significancia estadística de esta variable de control.

Tabla 5

Estimación de los parámetros de las variables de control

\begin{tabular}{lllll}
\hline $\ln ($ IVA $)$ & Coeficiente & Error estándar & Estadísticos t & Valor $\mathrm{p}$ \\
\hline $\mathrm{d} 1983$ & 5.459 & 0.20 & 27.83 & 0.000 \\
$\mathrm{~d} 1992$ & -0.681 & 0.14 & -4.91 & 0.000 \\
$\mathrm{~d} 1995$ & -0.226 & 0.15 & -1.51 & 0.132 \\
$\mathrm{~d} 2010$ & 1.112 & 0.14 & 8.06 & 0.000 \\
\hline
\end{tabular}

Fuente: elaboración propia.

\section{Conclusiones}

El tema de las finanzas públicas y la recaudación de impuestos es un tema con una relevancia significativa por la relación existente entre el Estado y los contribuyentes por la imposición de las contribuciones, a la fecha se tienen antecedentes de investigaciones relacionadas entre el comportamiento de la economía y los ingresos tributarios, específicamente lo relacionado al 
Producto Interno Bruto y al Impuesto al Valor Agregado. El estudio más relevante se denomina "Elasticidad ingreso de los impuestos federales en México efectos en la recaudación federal participable" escrito por Cárdenas, Ventosa y Gómez (2008), en el cual se concluyó que la elasticidad ingreso de la recaudación del IVA respecto del PIB es de 1.12, lo que significa que ante un aumento del $1 \%$ en el PIB la recaudación del IVA crecerá en 1.12\%.

A partir de este trabajo, se determinó que a mayor crecimiento económico se produce mayor recaudación del Impuesto al Valor Agregado. Este resultado parecería obvio dado que el PIB es el valor agregado de la economía y si aumenta necesariamente se debería generar mayor recaudación. El postulado teórico de la contabilidad del crecimiento establece que, si el impuesto a las ventas fuera homogéneo para todos los productos finales, esta relación sería constante y cada vez que se incremente el valor agregado de la economía también aumentará la recaudación del IVA. Sin embargo, en México por la presencia de tasas diferenciadas y exenciones el impuesto al valor agregado no es homogéneo y por ende existen distorsiones que hacen que la relación no sea constante; por lo tanto, se puede presentar una situación en la que los sectores de la economía que estén creciendo sean los que no están gravados con el IVA, situación, aunque poco probable es factible y que, de presentarse, la recaudación no se incrementaría.

Los resultados obtenidos en esta investigación concuerdan con las estimaciones realizadas en trabajos anteriores respecto de la relación directa entre el PIB y la recaudación del IVA; es decir, se confirma que a mayor crecimiento económico mayor recaudación del IVA. No obstante, los resultados obtenidos difieren en términos de magnitud respecto de las investigaciones anteriores ya que la elasticidad calculada es de 4.510 cuando en estudios previos la elasticidad oscila entre 1.12 y 1.88 . Es importante considerar que los periodos de estudio difieren y ésta puede ser la razón por la que las elasticidades son distintas.

Desde la óptica de los factores no económicos que influyen en la recaudación de impuestos se ubica el tema de la legislación tributaria y esta investigación siguiendo el modelo desarrollado por Katz y Bommarito (2014), realizó las estimaciones en cuanto al impacto de la complejidad legal sobre la recaudación del IVA.

Los resultados permiten afirmar que la relación entre la recaudación del IVA y la complejidad legal (estructura, lenguaje e interdependencia) es una relación inversa, lo que significa que realizar modificaciones legales para expresar al más mínimo detalle las diferentes interacciones que se presentan entre la autoridad tributaria y el contribuyente, pueden causar en términos de comprensión por parte de los sujetos obligados un efecto contraproducente a los objetivos originalmente trazados en las modificaciones realizadas, con el consecuente deterioro de la hacienda pública. 
La estimación del impacto de la complejidad legal sobre la recaudación del IVA se ubica en el mismo sentido de los diferentes teóricos que han investigado sobre el tema de complejidad en un texto legal, en específico de los que analizaron las leyes fiscales de los Estados Unidos; es decir, que a mayor complejidad se requiere de un mayor esfuerzo de análisis y comprensión de los textos legales, hasta el grado de requerir el apoyo de un experto en temas fiscales, con el consecuente incremento en los costos que impacta en el cumplimiento de las obligaciones incluyendo las obligaciones de pago.

La aportación de la presente investigación fue demostrar que, además de los efectos del crecimiento económico sobre la recaudación del IVA, la complejidad legal generada por las modificaciones al texto de la Ley tiene un impacto negativo sobre la recaudación del impuesto; es decir existe una relación inversa entre ambas variables. La elasticidad estimada para estas variables fue negativa (-1.139), lo que significa que ante el incremento de una unidad en el indicador de complejidad legal la recaudación disminuirá en $1.139 \%$.

Adicionalmente, esta investigación permitió identificar la factibilidad de extrapolar el uso de herramientas estadísticas robustas y de minería de texto para calcular la complejidad legal de cualquier otra Ley diferente de la Ley del IVA, de tal forma que, se pudiera disminuir la discrecionalidad en las modificaciones que se proponen al H. Congreso de la Unión a fin de mitigar los posibles efectos negativos que se generen por dichas modificaciones.

\section{Referencias}

ABA, (2018). American Bar Association. Estados Unidos: ABA. (Disponible en: https://www.americanbar.org/ news) y (Consultado: 18/04/2018)

Barbuta, N. (2011). A review of factors for tax compliance. Annals of “Dunarea de Jos” University of Galati. Fascicle I. Economics and Applied Informatics. Years XVII, 1/2011. (Disponible en: https://www.ann.ugal.ro/ eco) y (Consultado: 10/07/2018).

Barro, R. J. \& Sala-i-Martin, X. (2009). Crecimiento Económico. (1a. Ed.). Barcelona, España: Reverté. (Disponible en: https://www.amazon.es/Crecimiento-Econ\%C3\%B3mico-Robert-Barro/dp/8429126082) y (Consultado: 20/07/2018).

Barro, R. J. (1991). Economic growth in a cross section of countries. The quarterly journal of economics, 106(2), 407-443. https://doi.org/10.2307/2937943

Bernal, D., Padilla, M. y Pérez, V. (2011). Panorama de la Situación de los Contribuyentes en México. Procuraduría de la Defensa del Contribuyente. (Disponible en: http://www.prodecon.gob.mx/Documentos/PanoramaSituacionContribuyentesMexico31ago2011.pdf ) y (Consultado: 10/02/2018).

Bikas, E. y Andruskaite, E. (2013). Factors affecting value added tax revenue. 1st Annual International Interdisciplinary Conference, AIIC 2013, 24-26 April, Azores, Portugal Comisión Europea. (Disponible en: http:// eujournal.org/index.php/esj/article/viewFile/1230/1239) y (Consultado: 20/04/2018). 
Box, G. E. y Cox, D. R. (1964). An analysis of transformations. Journal of the Royal Statistical Society. Series B (Methodological), 26 (2), 211-252. (Disponible en: http://www.jstor.org/stable/2984418 ) y (Consultado: 20/07/2018).

Cárdenas, Ó., Ventosa-Santaulària, D. y Gómez, M. (2008). Elasticidad Ingreso de los Impuestos Federales en México: Efectos en la Recaudación Federal Participable. El Trimestre Económico, 75(2), 519-531. (Disponible en: http://www.jstor.org/stable/20857167) y (Consultado: 09/07/2018.

Castaño, E. (2011). Una estimación no paramétrica y robusta de la transformación Box-Cox para el modelo de regresión. Lecturas de economía, 75 (julio - diciembre, 2011), pp 89 - 106, Universidad de Antioquia. (Disponible en: http://hdl.handle.net/10495/3635 ) y (Consultado: 25/08/2018).

Centro de Estudios Económicos del Sector Privado, CEESP (2018). Actividad Económica, tomo III. Análisis Económico Ejecutivo, diciembre 2018, página 2. (Disponible en: http://ceesp.org.mx/wp-content/uploads/2018/12/AEJE_24Dic18.pdf) y (Consultado: 18/02/2018).

Centro de Estudios de las Finanzas Públicas, CEFP (2009). La elasticidad del ISR e IVA respecto al Producto Interno Bruto. Memoria de Cálculo, mcnotacefp/055/2009. Recuperado el 3 de febrero de 2016, de Cámara de Diputados. (Disponible en: http://www.cefp.gob.mx/notas/2009/notacefp0552009/mcnotacefp0552009.pdf) y (Consultado: 25/07/2018).

Comisión Económica para América Latina y el Caribe, CEPAL (2009). La economía de los ingresos tributarios. Un manual de estimaciones tributarias. (Fernando R. Martín, Ed.) Recuperado el enero de 2016, Instituto Latinoamericano y del Caribe de Planificación Económica y Social, Comisión Económica para América Latina y el Caribe. (Disponible en: http://www.cepal.org/ilpes/publicaciones/xml/1/37421/manual62.pdf) y (Consultado: 12/06/2018).

De la Fuente, A. (1997). Fiscal Policy and Growth in the OECD (No. 1755). CEPR Discussion Papers. (Disponible en: https://ideas.repec.org/p/cpr/ceprdp/1755.html) y (Consultado: 22/06/2018).

Diver, C. (1983). The Optimal Precision of Administrative Rules. The Yale Law Journal, 93(1), 65-109. (Disponible en: https://www.jstor.org/stable/796245) y (Consultado: 28/04/2018).

Easterly, W., \& Rebelo, S. (1993). Fiscal policy and economic growth. Journal of monetary economics, 32(3), 417458. https://doi.org/10.1016/0304-3932(93)90025-B

Elliot, B. E., Rothenberg, T. J., \& Stock, J. H. (1996). Efficient tests of the unit root hypothesis. Econometri$c a, 64(8), 13-36$. (Disponible en: https://www.econometricsociety.org/search/node/elliot ) y (Consultado: 25/06/2018)

Engen, E. M., \& Skinner, J. (1992). Fiscal policy and economic growth (No. w4223). National Bureau of Economic Research. (Disponible en: https://www.nber.org/papers/w4223) y (Consultado: 18/06/2018).

Engle, R. F., \& Granger, C. W. (1987). Co-integration and error correction: representation, estimation, and testing. Econometrica: journal of the Econometric Society, 251-276. (Disponible en: https://www.jstor.org/stable/1913236?seq=1\#page_scan_tab_contents) y (Consultado: 20/05/2018).

Givati, Y. (2009). Resolving Legal Uncertainty: The Fulfilled Promise of Advance Tax Rulings. Discussion Paper No. 30. Harvard Law School. Cambridge, MA. (Disponible en: http://www.law.harvard.edu/programs/ olin_center/fellows_papers/pdf/Givati_30.pdf) y (Consultado: 20/07/2018).

Gober, J. y Burns, J. (1997). The relationship between tax structures and economic indicators. Journal of International Accounting, Auditing and Taxation, 6(1), 1-24. https://doi.org/10.1016/S1061-9518(97)90010-0

Greene, W. H. (2018). Econometric Analysis. 8th Edition. Stern School of Business, New York University. Pearson. (Disponible en: https://www.pearson.com/us/higher-education/program/Greene-Econometric-Analysis-8th-Edition/PGM334862.html) y (Consultado: 20/07/2018).

Gujarati, D. y Porter, D. (2010). Econometría. Quinta Edición. Mc Graw Hill. (Disponible en: https://vdocuments. mx/econometria-damodar-n-gujarati-5ta-edpdf.html ) y (Consultado: 10/02/2018).

Gupta, A. (2007). Determinants of Tax Revenue Efforts in Developing Countries. International Monetary Fund. Working Paper WP707/184, July 2007. DOI: http://dx.doi.org/10.5089/9781451867480.001 
Helhel, Y. y Ahmed, Y. (2014). Factors affecting tax attitudes and tax compliance: a survey study in Yemen. European Journal of Business and Management, Vol 6, No. 22, 2014 pp 48-58. (Disponible en: https://www. researchgate.net/publication/270338082) y (Consultado: 10/04/2018).

Hinrichs, H. (1966). A General Theory of Tax Structure change during Economic Development. Harvard Law School International Tax Program Development. Record Number: 19661802098, pp 154. (Disponible en: https://www.cabdirect.org/cabdirect/abstract/19661802098) (Consultado: 08/06/2018).

Instituto Nacional de Estadística, Geografía e Informática (INEGI, 2018). Sistema de Cuentas Nacionales de México, Fuentes y Metodologías, Año base 2013. México, D. F. (Disponible en: https://www.inegi.org.mx/contenidos/programas/pibact/2013/metodologias/METODOLOGIA_CBYSB2013.pdf) y (Consultado: 15/07/2018).

Instituto Tecnológico y de Estudios Superiores de Monterrey ITESM (2013). Estudio de Evasión Global de Impuestos. Secretaría de Hacienda y Crédito Público, Servicio de Administración Tributaria, Administración General de Planeación. (Disponible en: http://omaww.sat.gob.mx/administracion_sat/estudios_evasion_fiscal/Paginas/ default.aspx) y (Consultado: 17/07/2018).

Johansen, S. (1988). Statistical analysis of cointegration vectors. Journal of economic dynamics and control, 12 (23), 231-254. https://doi.org/10.1016/0165-1889(88)90041-3

Kades, E. (1997). The Laws of Complexity and the Complexity of the Laws: The Implications of Computational Complexity Theory for the Law. William and Mary Law School, 49, 403-484. Faculty Publications. Paper 646. (Disponible en: https://scholarship.law.wm.edu/facpubs/646) y (Consultado: 25/03/2018).

Karagianni, S., Pempetzoglu, M. y Saraidaris, A. (2013). Average Tax Rates and Economic Growth: A Nonlinear Causality Investigation for the USA. Frontiers In Finance and Economics, 12(1), 51-59. (Disponible en: https://ssrn.com/abstract=2629248) y (Consultado:22/06/2018).

Katz, D. y M. Bommarito (2014). Measuring the complexity of the law: the United States Code. Michigan State University, 22 (4), 337 - 374. https://doi.org/10.1007/s10506-014-9160-8

King, R. G., \& Rebelo, S. (1990). Public policy and economic growth: developing neoclassical implications. Journal of Political Economy, 98(5), 126-150. https://doi.org/10.1086/261727

Kristoufek, L., Janda, K., \& Zilberman, D. (2014). Price transmission between biofuels, fuels, and food commodities. Biofuels, Bioproducts and Biorefining, 8(3), pp.362-373. (Disponible en: https://onlinelibrary.wiley.com/ doi/full/10.1002/bbb.1464 ) y (Consultado: 18/06/2018).

Kneller, R., Bleaney, M. F., \& Gemmell, N. (1999). Fiscal policy and growth: evidence from OECD countries. Journal of Public Economics, 74(2), 171-190. https://doi.org/10.1016/S0047-2727(99)00022-5

Koester, R. B., \& Kormendi, R. C. (1989). Taxation, aggregate activity and economic growth: cross-country evidence on some supply-side hypotheses. Economic Inquiry, 27(3), 367-386. https://doi.org/10.1111/j.1465-7295.1989. tb02011.x

Kormendi, R. C., \& Meguire, P. G. (1995). Government debt, government spending, and private-sector behavior: Reply. The American Economic Review, 85(5), 1357-1361. (Disponible en: https://www.jstor.org/stable/2950996?seq=1\#page_scan_tab_contents) y (Consultado:24/06/2018).

Leibfritz, W., Thornton, J., \& Bibbee, A. (1997). Taxation and economic performance. (Disponible en: https:// www.oecd-ilibrary.org/economics/taxation-and-economic-performance_668811115745) y (Consultado: 20/06/2018).

Levine, R., \& Renelt, D. (1992). A sensitivity analysis of cross-country growth regressions. The American Economic Review, 82(4), 942-963. (Disponible en: https://www.jstor.org/stable/2117352?seq=1\#page_scan_tab_contents) y (Consultado: 10/06/2018).

Mankiw, G. (2016). Macroeconomics. Ninth Edition, Harvard University, 2016. (Disponible en: http://www.academia.edu/35995441/Macroeconomics_9th_Edition_by_N.Gregory_Mankiw) y (Consultado: 18/06/2018).

Marsden, K. (1983). Links between Taxes and Economic Growth: Some empirical evidence. Staff Working Paper: no. SWP 605. Washington D.C. (Disponible en: http://documents.worldbank.org/curated/ en/447031468756620681/Links-between-taxes-and-economic-growth-some-empirical-evidence) y (Consultado: 10/06/2018). 
Mendoza, E. G., Milesi-Ferretti, G. M., \& Asea, P. (1997). On the ineffectiveness of tax policy in altering longrun growth: Harberger's superneutrality conjecture. Journal of Public Economics, 66(1), 99-126. https://doi. org/10.1016/S0047-2727(97)00011-X

Moreno, J., Beltrán, J. y Mata, L. (2017). Análisis Trimestral de la Complejidad Legal de la Ley del Impuesto al Valor Agregado en el periodo de 1978 a 2016. Panorama Económico, 12(24), 26-38. (Disponible en: http:// panoramaeconomico.mx/ojs/index.php/PE/article/view/162 ) y (Consultado: 16/04/2018).

Musgrave, R. (1969). Fiscal Systems. Studies in Comparative Economies, No. 10. New Haven and London, Yale University Press, 1969, XIX p. 397., paper 27. https://doi.org/10.1017/S0770451800025124

Organización para la Cooperación y el Desarrollo Económicos, OCDE. (2004). Compliance Risk Management: Managing and Improving Tax Compliance. Committee on Fiscal Affairs. October 2004. Organization for Economic Cooperation and Development. (disponible en: https://www.oecd.org/tax/administration/33818656.pdf) y (Consultado: 04/02/2016).

Parkin, M y Loria E. (2015). Macroeconomía versión para Lationoamérica. Décimo primera edición. Editorial Pearson. (Disponible en: http://www.mypearsonshop.com.mx/mexico/tienda-online/economia-parkin-11ed-ebook1) (Consultado: 25/07/2018).

Plosser, C. I. (1992, August). The search for growth. In A Symposium Sponsored By The Federal Reserve Bank Of Kansas City, Policies For Long-Run Economic Growth (pp. 57-86). (Disponible en: http://citeseerx.ist.psu.edu/ viewdoc/download?doi=10.1.1.450.6478\&rep=rep1\&type=pdf\#page=93) y (Consultado: 28/05/2018).

Porunov, A. (2010). Mathcad in Hands of the Economist: Box-Пox Transformation and the Illusion of «Normality» of Macroeconomic Series. Biznes-informatika, 2, pp. 3-10. (Disponible en: https://bijournal.hse.ru/en/2010-2/26645455.html ) y (Consultado: 10/06/2018).

Ruhl, J. y Katz, D. (2015). Measuring, monitoring and managing legal complexity. Iowa Law Review, volume101, issue 1, 191-244. (Disponible en: https://ilr.law.uiowa.edu/print/volume-101-issue-1/measuring-monitoring-and-managing-legal-complexity/) y (Consultado: 20/03/2018).

Sacasa, M. (2012). Elasticidad ingreso de los impuestos y cambio climático. Finanzas Públicas y Cambio Climático en México (págs. 89-98). Programa de Naciones Unidas para el Desarrollo. PNUD. (Disponible en: http:// www.mx.undp.org/content/dam/mexico/docs/Publicaciones/MedioAmbientePublicaciones/UNDP-MX-Environ-FiPublicasyCCenMX-2012.pdf) y (Consultado: 26/06/2018).

Sakia, R. M. (1992). The Box-Cox Transformation Technique: A Review. Journal of the Royal Statistical Society. Series D (The Statistician). Vol. 41, No. 2 (1992), pp 169-178. DOI: 10.2307/2348250 (Disponible en: https:// www.jstor.org/stable/2348250 ) y (Consultado: 17/06/2018).

Schuck, P. (1992). Legal Complexity: Some Causes, Consequences, and Cures. Duke Law Journal, 42(1), 1-52. (Disponible en: https://scholarship.law.duke.edu/dlj/vol42/iss1/1) y (Consultado: 25/04/2018).

Slemrod, J., \& Yitzhaki, S. (1995). The costs of taxation and the marginal cost of funds (No. 95/83). International Monetary Fund. (Disponible en: https://ideas.repec.org/p/imf/imfwpa/95-83.html) y (Consultado: 30/06/2018).

Solow, R. M. (1956). A contribution to the theory of economic growth. The Quarterly Journal of Economics, 70(1), 65-94. https://doi.org/10.2307/1884513

Surrey, S. (1969). Complexity and the Internal Revenue Code: The Problem of the Management of Tax Detail. Law and Contemporary Problems. Vol. 34, No. 4, Tax Simplification and Reform (Autumm, 1969), pp. 673-710. DOI: 10.2307/1190905 (Disponible en: https://www.jstor.org/stable/1190905) y (Consultado: 10/03/2018).

Vélez, J., Correa, J.C. y Marmolejo-Ramos, F. (2015). A new approach to the Box-Cox transformation. Frontiers in Applied Mathematics and Statistics, 1(12), pp. 1-10. (Disponible en: https://www.frontiersin.org/articles/10.3389/fams.2015.00012/full ) y (Consultado: 30/07/2018).

Wright, R. (1996). Taxes, redistribution, and growth. Journal of Public Economics, 62(3), 327-338. https://doi. org/10.1016/0047-2727(95)01570-1 